\title{
Environmental Risk Factors and Amyotrophic Lateral Sclerosis: An Umbrella Review and Critical Assessment of Current Evidence from Systematic Reviews and Meta-Analyses of Observational Studies
}

\author{
Lazaros Belbasis $^{\mathrm{a}}$ Vanesa Bellou ${ }^{\mathrm{a}}$ Evangelos Evangelou ${ }^{\mathrm{a}} \mathrm{b}$ \\ a Department of Hygiene and Epidemiology, University of loannina Medical School, loannina, Greece; \\ ${ }^{b}$ Department of Biostatistics and Epidemiology, Imperial College London, London, UK
}

\section{Key Words}

Amyotrophic lateral sclerosis · Risk factors · Epidemiology ·

Meta-analysis · Umbrella review

\begin{abstract}
Background: The pathogenesis of amyotrophic lateral sclerosis (ALS) involves both environmental and genetic factors. Our study aimed at summarising the environmental risk factors for ALS, assessing the evidence for diverse biases, and pinpointing risk factors with high epidemiological credibility. Methods: We searched PubMed from inception to August 20,2015 , to identify systematic reviews and meta-analyses of observational studies examining associations between environmental factors and ALS. For each meta-analysis, we estimated the summary effect size by the use of randomeffects and fixed-effects models, the $95 \% \mathrm{Cl}$, the $95 \%$ prediction interval (PI), and the between-study heterogeneity. We assessed the evidence of small-study effects and excess significance bias. Results: Sixteen unique meta-analyses of different risk factors and ALS were considered. Of them, 5 were statistically significant at $p<0.001$ under the random-effects model. Only one factor presented robust evidence for a convincing association. This association pertained to chronic oc-
\end{abstract}

cupational exposure to lead (random-effects OR $1.81,95 \% \mathrm{Cl}$ 1.39-2.35). Conclusions: A small number of published metaanalyses on environmental factors and risk of ALS was identified, a phenomenon that could be attributed to the challenges in studying a rare neurological disease. More observational studies with adequate sample size and study design are needed to clarify the environmental component of ALS pathogenesis.

(c) 2016 S. Karger AG, Basel

\section{Introduction}

Amyotrophic lateral sclerosis (ALS) is a rapidly progressive, neurodegenerative disorder that affects motor neurons in brain, brainstem and spinal cord [1]. A majority of ALS cases are sporadic with unknown aetiology, whereas about $10 \%$ are familial with dominant inheritance [2]. In Europe, the incidence of ALS is about 2 per 100,000 [3], the prevalence of ALS is 5.40 per 100,000 [4] and the overall lifetime risk of developing ALS is 1:350 for men and 1:400 for women [5]. Although there is a clear genetic predisposition to ALS [1], environmental factors are likely to be involved in the pathogenesis of the disease.

\section{KARGER}

E-Mail karger@karger.com

www.karger.com/ned
(C) 2016 S. Karger AG, Base

0251-5350/16/0462-0096\$39.50/0
Dr. Evangelos Evangelou, PhD

Department of Hygiene and Epidemiology

University of Ioannina Medical School

GR-45110 Ioannina (Greece)

E-Mail vangelis@ cc.uoi.gr 
Environmental exposures proposed to be linked with ALS include exposure to lead and other heavy metals, and pesticides, while medical conditions including head trauma have also been associated with ALS [1]. Clarifying the environmental component of ALS etiology could lead to better understanding of disease pathophysiology. Due to rapid accumulation of observational studies, systematic reviews accompanied by a quantitative synthesis of the available evidence are the most appropriate approaches to identify credible associations and to systematically appraise the observational studies.

Previous umbrella reviews of systematic reviews and meta-analyses that assessed environmental factors associated with Parkinson's disease and multiple sclerosis revealed several caveats pertaining to large statistical between-study heterogeneity, small sample sizes, and smallstudy effects and other biases [6,7]. Out of 44 associations, only 3 showed strong evidence for association with multiple sclerosis [6], whereas for Parkinson's disease, only 2 of the examined environmental factors achieved top ranking in the assessment of the meta-analyses [7]. All these methodological issues introduce doubts for the epidemiological credibility of several associations between the environmental factors and the aforementioned neurological diseases.

To further expand the mapping and the critical appraisal of environmental risk factors across additional neurological conditions, we apply similar methodology for ALS. Our aim is to provide an overview of the range and validity of the published associations of diverse environmental risk factors with ALS as examined in published systematic reviews and meta-analyses, and to evaluate the existence and the extent of potential biases in this literature. Finally, we highlight which of the previously studied associations that have been synthesized with meta-analyses have the strongest evidence for association.

\section{Methods}

\section{Search Strategy and Eligibility Criteria}

We conducted an umbrella review, a systematic collection and assessment of multiple systematic reviews and meta-analyses performed on a specific research topic [8]. The methods of the umbrella review are standardized and in this work we follow state-ofthe-art approaches as previously published umbrella reviews on risk factors for various outcomes including multiple sclerosis and Parkinson's disease $[6,7]$. Specifically, our approach includes a comprehensive and systematic literature search, application of state-of-the art methods for the evaluation of potential biases, and assessment of the epidemiological credibility of the examined associations by predefined criteria.

Environmental Risk Factors and ALS
We systematically searched PubMed from inception to August 20,2015 to identify systematic reviews and meta-analyses of observational studies examining associations between environmental (non-genetic) factors and ALS. The search strategy used the keywords ('amyotrophic lateral sclerosis' OR 'motor neuron disease') AND ('systematic review' OR 'meta-analysis'). The full text of potentially eligible articles was scrutinised independently by 2 investigators (L.B. and V.B.). We excluded meta-analyses that investigated the association between genetic polymorphisms and risk of ALS because these factors are already summarized and evaluated in ALS Gene (http://www.alsgene.org/) [9]. We excluded metaanalyses examining environmental risk factors for other motor neuron diseases than ALS. We also excluded meta-analyses that examined ALS as a risk factor for other medical conditions. We did not apply any language restrictions in the selection of eligible studies. When more than one meta-analysis on the same research question was eligible, the meta-analysis with the largest number of component studies with data on individual studies' effect sizes was retained for the main analysis. We enhanced the literature search by accessing additional databases (the Cochrane Library and EMBASE).

\section{Data Extraction}

Data extraction was conducted by 2 independent investigators (L.B. and V.B.). In case of discrepancies, the final decision was made by a third investigator (E.E.). From each eligible article, we extracted information on the first author, journal, year of publication, examined risk factors, and number of studies included. We also recorded the number of cases and controls in each study for each risk factor. When the sample sizes of the primary studies were not reported in the article of meta-analysis, we retrieved the published report of the primary study and we extracted the relevant data. If a quantitative synthesis was performed, we also recorded the study-specific relative risk estimates (risk ratio (RR), odds radio (OR), hazard ratio (HR)) along with the corresponding CI.

\section{Statistical Analysis}

For each meta-analysis, we estimated the summary effect size and its 95\% CI with both fixed-effects and random-effects models $[10,11]$. We also estimated the $95 \%$ PI, which further accounts for between-study heterogeneity and evaluated the uncertainty for the effect that would be expected in a new study addressing that same association $[12,13]$. In the case of meta-analyses with continuous data, the effect estimate was transformed to an OR with an established formula [14]. For the largest study of each meta-analysis, we estimated the SE of the effect size and examined whether the SE was less than 0.10 . In a study with an SE of less than 0.10 , the difference between the effect estimate and the upper or lower $95 \% \mathrm{CI}$ is less than 0.20 (i.e. this uncertainty is less than what is considered a small effect size).

Between-study heterogeneity was assessed and quantified by the $\mathrm{I}^{2}$ metric [15]. $\mathrm{I}^{2}$ ranges between 0 and $100 \%$ and describes the percentage of total variation across studies that is due to heterogeneity rather than chance [16]. Values exceeding 50 or $75 \%$ are usually judged to represent large or very large heterogeneity, respectively.

We applied the regression asymmetry test proposed by Egger and colleagues [17] in order to assess whether there was evidence for small-study effects (i.e. whether smaller studies tend to give substantially larger estimates of effect size compared with larger 
Fig. 1. Flowchart of literature search for systematic reviews and meta-analyses published from inception until August 20, 2015.

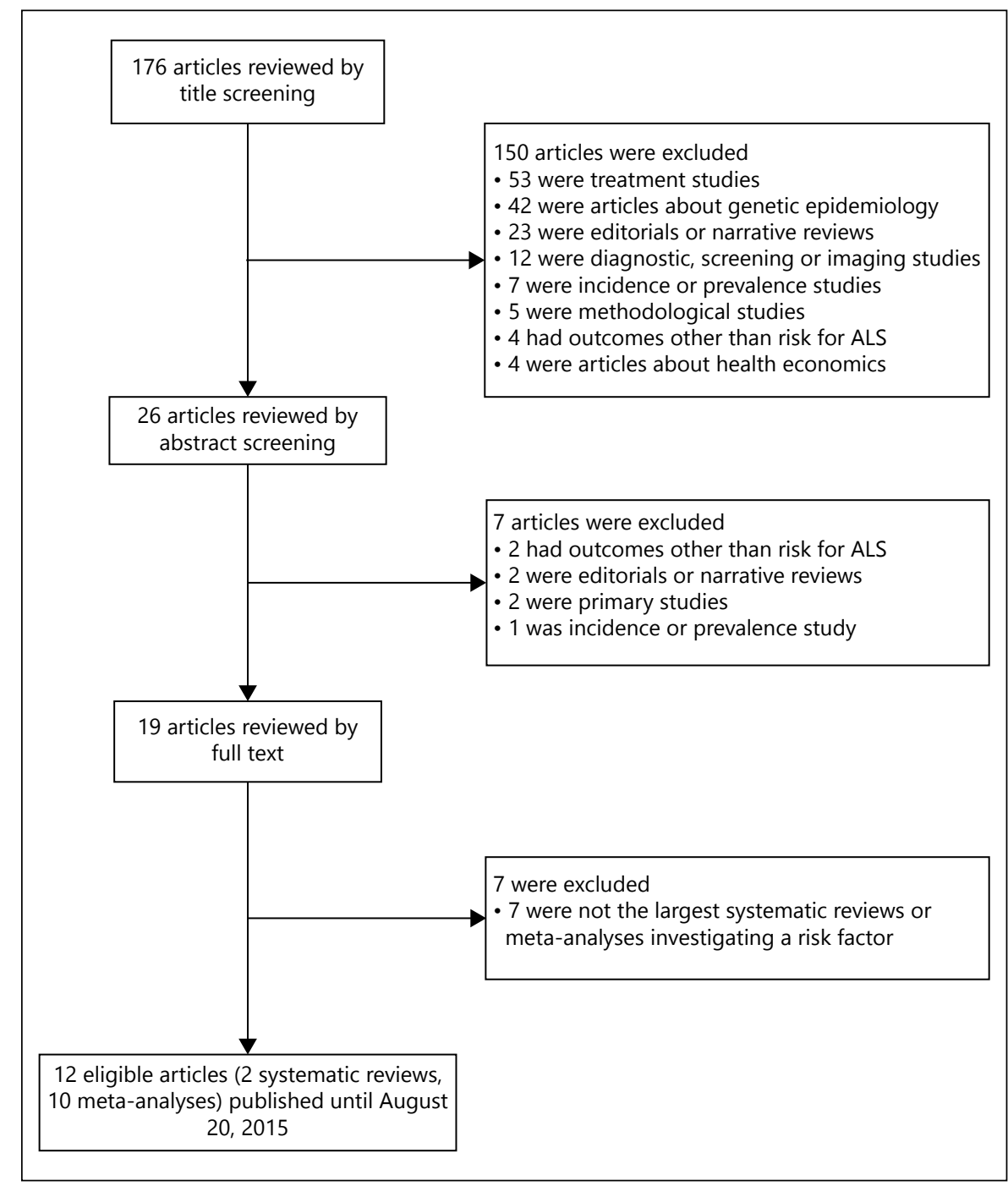

studies). A $\mathrm{p}<0.10$ accompanied by a more conservative effect in larger studies than in random-effects meta-analysis was judged to be evidence for the existence of small-study effects.

We also applied the excess statistical significance test, which evaluates whether the observed $(\mathrm{O})$ number of studies with nominally statistically significant results ('positive' studies, $\mathrm{p}<0.05$ ) is larger than their expected (E) number [18]. E is calculated in each meta-analysis by the sum of the statistical power estimates for each component study. The true effect size for any meta-analysis is not known. We estimated the power of each component study using the effect size of the largest study (smallest SE) in a meta-analysis [19]. The power of each study was calculated using a non-central $t$ distribution [20]. Excess statistical significance for single metaanalyses was claimed at 2 -sided $\mathrm{p}<0.10$ with $\mathrm{O}>\mathrm{E}$ as previously proposed.

Finally, we identified and reported the associations that had the strongest validity and were not suggestive of bias. Specifically, we characterized as convincing the associations that met the following criteria: had significance according to random-effects meta-anal- ysis less than $0.001[21,22]$; were based on greater than 1,000 cases; had between-study heterogeneity that was not large $\left(\mathrm{I}^{2}<50 \%\right)$ and a 95\% PI excluded the null value; and had not evidence of small-study effects and excess significance bias. We characterized as suggestive the associations that presented not large betweenstudy heterogeneity, and had not evidence of small-study effects and excess significance bias. The remaining significant associations were characterized as weak.

The statistical analysis and the power calculations were performed with STATA version 12.0.

\section{Results}

Our literature search on PubMed yielded 176 articles, and 12 articles were deemed eligible (fig. 1). The additional literature search on the Cochrane Library and 
EMBASE did not result in any further eligible articles. Two systematic reviews $[23,24]$ did not quantify the existent evidence, while 10 articles additionally performed a meta-analysis. These articles corresponded to 16 unique meta-analyses, examining 16 environmental factors and including 138 primary observational studies in total. One paper [25] included 2 meta-analyses on the association between smoking and ALS, providing analyses by 2 different level of comparisons (ever smokers vs. never smokers, and current smokers vs. never smokers). In our study, we included the meta-analysis that compared the difference in risk between ever smokers and never smokers, as this analysis included more ALS cases and more observational studies. Seven of the 19 articles screened by full text were excluded because a larger meta-analysis that examined the same risk factors was found. These articles pertained to extremely-low-frequency electromagnetic fields [26], smoking [27], pesticides [28-30], and heavy metals $[29,31]$.

The 2 systematic reviews of observational studies without further quantitative synthesis examined physical activity [24] (37 studies) and diabetes mellitus [23] (7 studies) as risk factors for ALS. In both cases, the authors concluded that the evidence is limited and quite heterogeneous, and could not be used to draw definite conclusions. In both systematic reviews, the authors mentioned that a quantitative synthesis was not attempted because of heterogeneity in study designs, in ascertainment and definition of the exposure.

The median number of studies per meta-analysis was 8 (IQR 5-9) and the median number of cases was 1,779 (IQR 937-2,911). The 16 meta-analyses covered a range of risk factors, classified as biomarkers $(n=5)$, dietary factors $(n=2)$, exposure to toxic agents $(n=5)$ and medical history $(n=2)$. The number of cases was greater than 1,000 in 11 meta-analyses. All eligible meta-analyses used summary-level data from published literature and none of them had access to individual participant data.

Nine of 16 meta-analyses reported effects that were significant at $\mathrm{p}<0.05$ under the random-effects model. Five were significant at $\mathrm{p}<0.001$ under the randomeffects model (table 1): farming [32], lead [33], n-3 fatty acids [34], pesticides [32] and serum uric acid [35]. The heterogeneity was large ( $\mathrm{I}^{2} \geq 50$ and $\left.\leq 75 \%\right)$ in 5 metaanalyses and very large $\left(\mathrm{I}^{2}>75 \%\right)$ in 3 meta-analyses. The meta-analyses with very large heterogeneity examined serum total cholesterol [36], serum HDL [36] and serum LDL [36]. Only 2 meta-analyses had a 95\% PI excluding the null value, pertaining to lead and n-3 fatty acids. In 8 of the eligible meta-analyses, the

Environmental Risk Factors and ALS
SE of the largest study was less than 0.10 in a $\log$ OR scale.

Two meta-analyses presented statistically significant Egger's test $(p<0.10)$. In one of these 2 meta-analyses, the largest individual study had a more conservative effect size than the random-effects summary effect size, indicating the presence of small-study effects. Assuming that the effect size in the largest study was the true effect, 5 of the 16 meta-analyses had a significant difference $(\mathrm{p}<0.10)$ between the number of observed and expected positive studies (table 2). These meta-analyses pertained to serum total cholesterol, serum HDL, serum LDL, statins and pesticides.

By applying our credibility criteria, 7 out of the 16 eligible environmental factors presented a non-significant association ( $p>0.05)$. Of the 9 remaining significant associations at $\mathrm{p}<0.05,2$ associations (extremely low-frequency electromagnetic fields, serum uric acid) presented large heterogeneity and one association ( $n-3$ fatty-acids intake) was significant for small-study effects and an additional association (pesticides) presented hints for excess statistical significance. Thus, the evidence of association for those 4 factors was weak. Of the remaining 5 associations, 4 (other heavy metals, farming, head injury, $\beta$-carotene) had 95\% PI including the null value, whereas one association (other heavy metals) included less than 1,000 cases. These 4 associations were supported by suggestive evidence.

Finally, only one of the 16 meta-analyses (exposure to lead [33]) presented convincing evidence for an association with ALS, supported by more than 1,000 cases, $\mathrm{p}<$ 0.001 under the random-effects model, small or moderate heterogeneity $\left(\mathrm{I}^{2}<50 \%\right)$, a 95\% PI excluding the null value, absence of evidence for small-study effects and excess statistical significance. This association had a summary OR of $1.81\left(95 \%\right.$ CI $\left.1.39-2.35 ; \mathrm{p}=2.13 \times 10^{-6}\right)$ with small heterogeneity $\left(\mathrm{I}^{2}=12.7 \%\right)$. Evidence was supported by a total of 1,228 cases. An overall assessment of significant risk factors for ALS, using the predefined criteria based on the sample size, the level of replication, a more conservative threshold for $\mathrm{p}<0.001$, and the presence of smallstudy effects, is presented in table 3 .

\section{Discussion}

We present an overview and critical appraisal of environmental risk factors that have been associated with ALS in published systematic reviews and meta-analyses, by applying specific methodological criteria. To our knowl- 


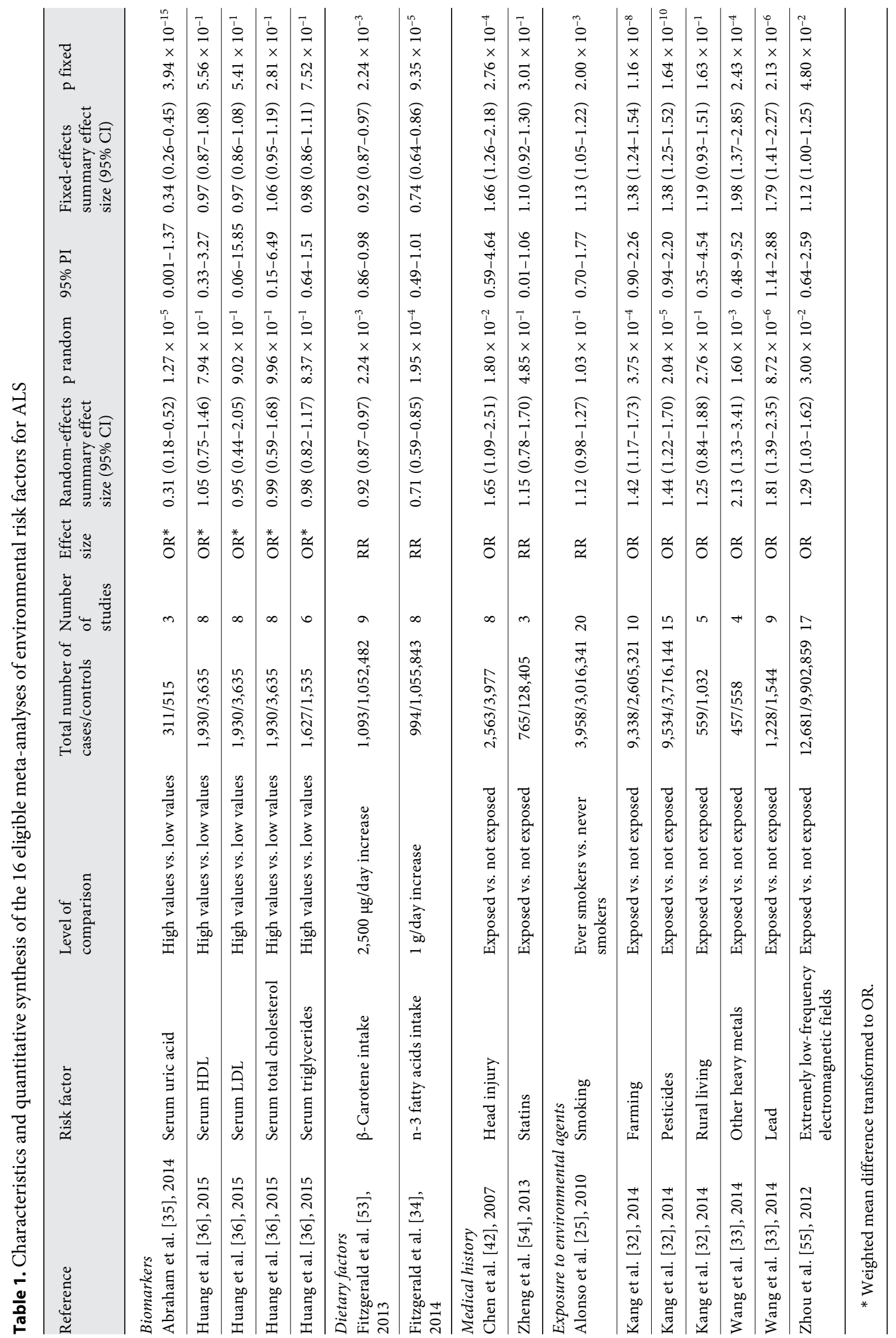




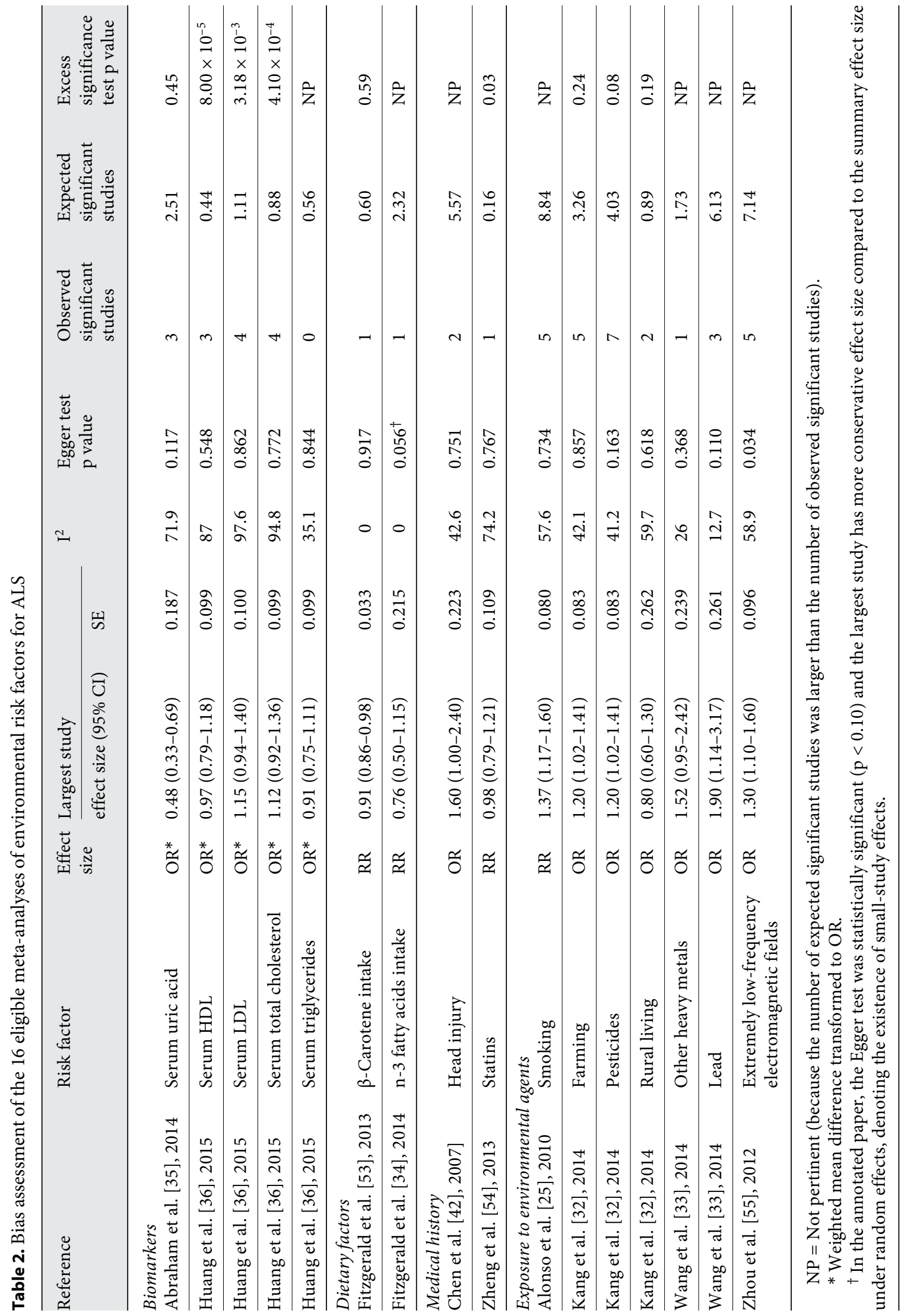




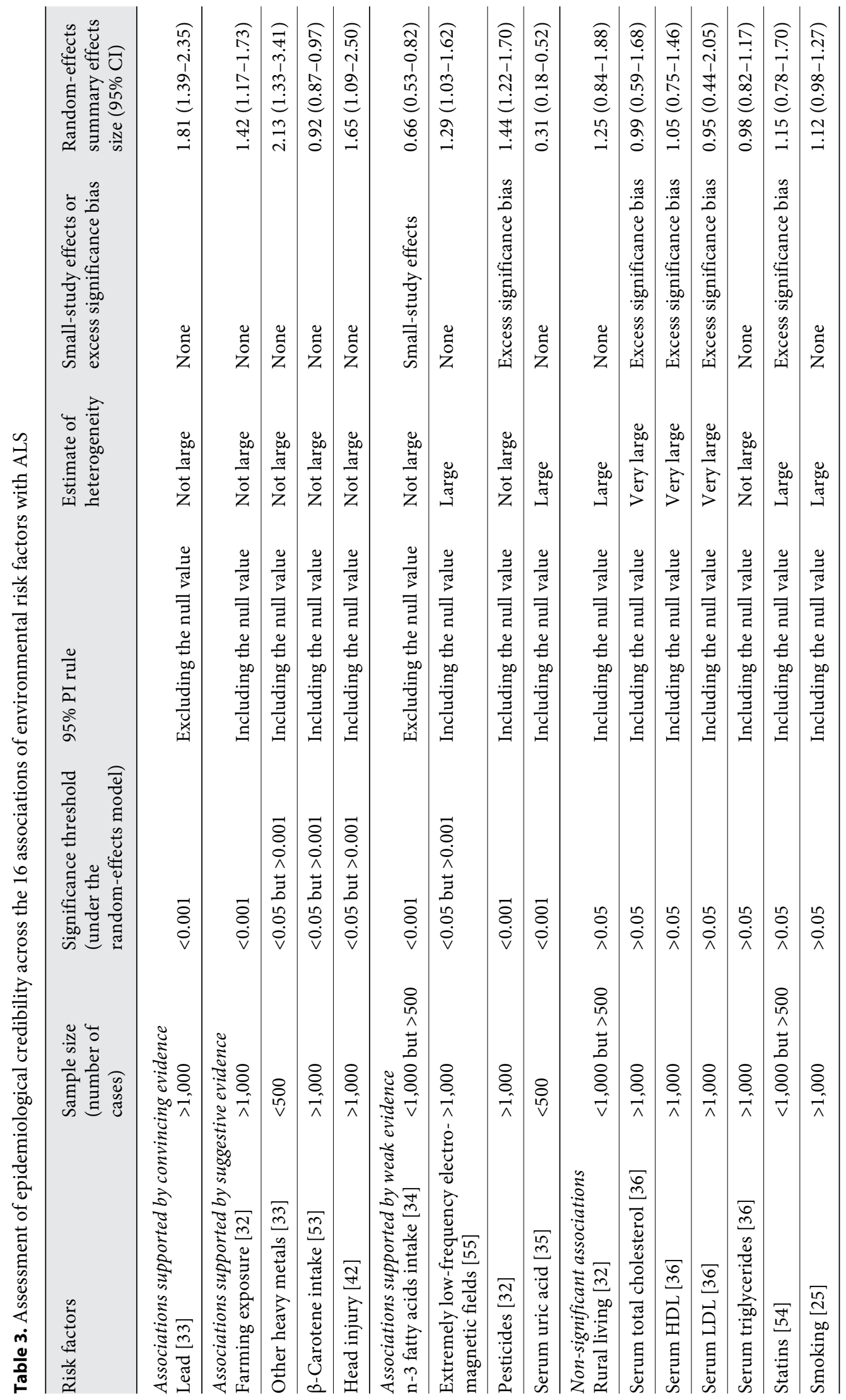


edge, this is the first attempt to summarize the evidence from existing meta-analyses on environmental factors for ALS, and their limitations. Juxtaposing other umbrella reviews $[6,37]$ and field synopses of genetic associations of complex diseases [38], an assessment of environmental factors, especially for rare diseases, could be rather challenging due to biases derived from the study design, small sample sizes, and misclassification problems mainly for the exposure and so on. A stringent, comprehensive appraisal of current evidence is necessary to minimize false positive associations.

In our study, we observed a small number of published meta-analyses synthesizing the evidence on environmental factors and risk of ALS. This could be attributed to the small number of observational studies due to the difficulties of conducting research focusing on a rare neurological disease (e.g. the vast number of environmental exposures that can be studied, the large cost of conducting population-based studies combined with small interest of funding bodies for a rare disease) [5]. Overall, 16 risk factors have been studied in a meta-analysis for an association with the disease, including biomarkers, dietary factors, exposure to toxic agents and medical history. However, only one of these factors, chronic occupational exposure to lead, was supported by strong epidemiological credibility, as expressed by large sample size, not large heterogeneity, absence of small-study effects and excess statistical significance, a $\mathrm{p}<0.001$, and a 95\% PI excluding the null value.

The effect size and the magnitude of the association between chronic occupational exposure to lead and risk for ALS were strong. Indeed, the predominant theory on the environmental counterpart to ALS pathogenesis is based on lead toxicity [1]. In humans, the classic form of lead neuropathy is characterized by ALS-like clinical manifestations, such as weakness initially involving the wrist and finger extensors and with spread to other muscles [33]. Additionally, several studies measured biomarkers of lead exposure in ALS patients and they reported higher levels of lead in blood for ALS patients compared to healthy controls $[39,40]$. However, the observational studies on the association between lead exposure and risk for ALS included a self-reported assessment of occupational exposure to lead [41]. To clarify the association between lead and risk of ALS, it is apparent that more epidemiological studies are needed to assess the exposure to lead by biomarkers on biological fluids. Exposure to other heavy metals, such as mercury and aluminum that has also been proposed to be associated with risk for ALS [1], was supported by suggestive evidence,

Environmental Risk Factors and ALS indicating that there is no solid body of evidence to underpin this association.

Furthermore, head injury presented a positive association with ALS, supported by suggestive evidence and a $\mathrm{p}$ value close to nominal significance threshold of 0.05 . This association has been argued to have biological plausibility, and several mechanisms, that apply to other neurological diseases as well, have been proposed to explain the neurological damage induced by head injury [42, 43]. However, as in the case of Parkinson's disease [7, 44], the association between head injury and ALS may be attributed to inverse causation, and it may be perceived as a prodromal manifestation of ALS. To draw a definite conclusion on the relation between head injury and ALS, we should take into account the time lap between the event of head injury and the clinical manifestation or diagnosis of ALS. Although the largest study reported a statistically significant effect, the observational studies were characterized by poor reporting on the assessment of exposure, small sample sizes and low methodological quality [42, 45], while they did not perform adequate adjustments for potential confounders. The low statistical power and the existence of potential confounders introduce doubts for the credibility of the association between head injury and ALS.

As expected, a majority of meta-analyses on the association of biomarkers with risk for ALS had large or very large between-study heterogeneity and, in several cases, hints suggestive for excess statistical significance. A similar picture was also observed in the field of multiple sclerosis [6] and Parkinson's disease [7]. The identification of credible biomarkers for ALS is essential for diagnosis and prognosis of ALS, and the development of novel biomarkers could even alter the design of clinical trials in the field of ALS [3]. However, the respondent meta-analyses presented evidence for various statistical biases, verifying the absence of any plausible biomarker. Previous research in other fields highlighted that the lack of replication, the large uncertainty in the effect sizes and the existence of statistical biases are a common phenomenon in the literature of biomarkers, indicating the poor design of the observational studies [46, 47].

Regarding the analysis of bias, one third of the examined meta-analyses were characterized by the presence of excess statistical significance. Compared to the previously published umbrella reviews, the level of excess statistical significance is higher than the case of multiple sclerosis but quite smaller than the case of Parkinson's disease $[6,7]$. Also, a large proportion of the examined metaanalyses was poorly replicated, given that half of the me- 
ta-analyses had large or very large heterogeneity, and only 2 of the meta-analyses presented a 95\% PI excluding the null value. The level of replication is comparable with that in the case of multiple sclerosis and Parkinson's disease $[6,7]$. Heterogeneity might often be a manifestation of bias in some studies of a meta-analysis, but could also emerge from genuine differences across studies. Potential sources of heterogeneity might be the mixture of cohort studies and case-control studies in some of the metaanalyses, and differences in exposure assessment.

Our analysis has some caveats. First, both asymmetry and excess statistical significance tests offer suggestions of bias, and not definitive proof thereof. Second, effect inflation might affect even the results of the largest studies because often these studies do not have an adequate sample size and appropriate study design or might have had inherent biases themselves. Thus, our estimates of the extent of excess statistical significance are probably conservative. Additionally, we did not appraise the quality of the individual component primary studies because this was beyond the scope of this umbrella review. This was the aim of the original systematic reviews and meta-analyses, which should include an assessment of study quality and examine whether the study should be included in the quantitative calculations. Also, in our analysis, we assessed only associations considered by meta-analyses of observational studies. Thus, we might miss other associations supported by adequate evidence that have not yet been assessed through meta-analytic approaches.

Acknowledging these caveats, our assessment depicts the status of evidence on 16 associations between environmental factors and risk for ALS. Only chronic occupational exposure to lead provided credible evidence for positive association with ALS without the presence of substantial caveats. Our approach could be complemented by the application of existing evaluation tools focused on the methodological appraisal of systematic reviews, such as AMSTAR [48, 49]. Data from more studies and investigation of sources of heterogeneity are needed to better understand the associations between the remaining risk factors and ALS. As previously suggested, for observational research and biomarker studies in general, use of standardized definitions for outcomes and exposures, adoption of reporting guidelines (e.g. Strengthening the Reporting of Observational Studies in Epidemiology (STROBE) ) [50], and registration of hypothesis-testing observational studies might help to improve the evidence in the future $[51,52]$.

\section{Acknowledgement}

There was no funding source for this study. All authors had full access to all the study data. The corresponding author had final responsibility for the decision to submit for publication.

\section{Author Contributions}

L.B. and V.B. had the original idea for the manuscript and all authors contributed to design the study. L.B., V.B. performed the analyses and all authors interpreted the results. L.B., V.B. wrote the first draft of the manuscript. All authors critically reviewed, wrote and approved the final version.

\section{Disclosure Statement}

The authors declare that there are no conflicts of interest.

\section{References}

1 Paez-Colasante X, Figueroa-Romero C, Sakowski S, Goutman S, Feldman EL: Amyotrophic lateral sclerosis: mechanisms and therapeutics in the epigenomic era. Nat Rev Neurol 2015;11:266-279.

2 Renton AE, Chiò A, Traynor BJ: State of play in amyotrophic lateral sclerosis genetics. Nat Neurosci 2014;17:17-23.

3 Kiernan MC, Vucic S, Cheah BC, Turner MR, Eisen A, Hardiman O, et al: Amyotrophic lateral sclerosis. Lancet 2011;377:942-955.

4 Chiò A, Logroscino G, Traynor BJ, Collins J, Simeone JC, Goldstein LA, et al: Global epidemiology of amyotrophic lateral sclerosis: a systematic review of the published literature. Neuroepidemiology 2013;41:118-130.
5 Al-Chalabi A, Hardiman O: The epidemiology of ALS: a conspiracy of genes, environment and time. Nat Rev Neurol 2013;9:617-628.

6 Belbasis L, Bellou V, Evangelou E, Ioannidis JP, Tzoulaki I: Environmental risk factors and multiple sclerosis: an umbrella review of systematic reviews and meta-analyses. Lancet Neurol 2015; 14:263-273.

7 Bellou V, Belbasis L, Tzoulaki I, Evangelou E, Ioannidis JP: Environmental risk factors and Parkinson's disease: an umbrella review of meta-analyses. Parkinsonism Relat Disord 2015; Inpress,DOI:10.1016/j.parkreldis.2015.12.008.

8 Ioannidis JP: Integration of evidence from multiple meta-analyses: a primer on umbrella reviews, treatment networks and multiple treatments meta-analyses. CMAJ 2009;181: 488-493.

9 Lill CM, Abel O, Bertram L, Al-Chalabi A: Keeping up with genetic discoveries in amyotrophic lateral sclerosis: the ALSoD and ALSGene databases. Amyotroph Lateral Scler 2011;12:238-249.

10 DerSimonian R, Laird N: Meta-analysis in clinical trials. Control Clin Trials 1986;7:177188.

11 Lau J, Ioannidis JP, Schmid CH: Quantitative synthesis in systematic reviews. Ann Intern Med 1997;127:820-826.

12 Higgins JP, Thompson SG, Spiegelhalter DJ: A re-evaluation of random-effects meta-analysis. J R Stat Soc Ser A Stat Soc 2009;172:137-159. 
13 Higgins JP: Commentary: heterogeneity in meta-analysis should be expected and appropriately quantified. Int J Epidemiol 2008;37: 1158-1160.

14 Chinn S: A simple method for converting an odds ratio to effect size for use in meta-analysis. Stat Med 2000;19:3127-3131.

15 Cochran WG: The combination of estimates from different experiments. Biometrics 1954; 10:101-129.

16 Higgins JP, Thompson SG: Quantifying heterogeneity in a meta-analysis. Stat Med 2002; 21:1539-1558.

17 Egger M, Davey Smith G, Schneider M, Minder C: Bias in meta-analysis detected by a simple, graphical test. BMJ 1997;315:629-634.

18 Ioannidis JP, Trikalinos TA: An exploratory test for an excess of significant findings. Clin Trials 2007;4:245-253.

19 Ioannidis JP: Clarifications on the application and interpretation of the test for excess significance and its extensions. J Math Psychol 2013;57:184-187.

20 Lubin JH, Gail MH: On power and sample size for studying features of the relative odds of disease. Am J Epidemiol 1990;131:552566.

21 Ioannidis JP, Tarone R, McLaughlin JK: The false-positive to false-negative ratio in epidemiologic studies. Epidemiology 2011;22:450456.

22 Johnson VE: Revised standards for statistical evidence. Proc Natl Acad Sci U S A 2013;110: 19313-19317.

23 Lekoubou A, Matsha TE, Sobngwi E, Kengne AP: Effects of diabetes mellitus on amyotrophic lateral sclerosis: a systematic review. BMC Res Notes 2014;7:171.

24 Hamidou B, Couratier P, Besançon C, Nicol M, Preux PM, Marin B: Epidemiological evidence that physical activity is not a risk factor for ALS. Eur J Epidemiol 2014;29:459-475.

25 Alonso A, Logroscino G, Hernán MA: Smoking and the risk of amyotrophic lateral sclerosis: a systematic review and meta-analysis. J Neurol Neurosurg Psychiatry 2010;81:12491252.

26 Vergara X, Kheifets L, Greenland S, Oksuzyan S, Cho YS, Mezei G: Occupational exposure to extremely low-frequency magnetic fields and neurodegenerative disease: a meta-analysis. J Occup Environ Med 2013;55:135-146.

27 Wang H, O'Reilly ÉJ, Weisskopf MG, Logroscino G, McCullough ML, Thun MJ, et al: Smoking and risk of amyotrophic lateral sclerosis: a pooled analysis of 5 prospective cohorts. Arch Neurol 2011;68:207-213.

28 Malek AM, Barchowsky A, Bowser R, Youk A, Talbott EO: Pesticide exposure as a risk factor for amyotrophic lateral sclerosis: a meta-analysis of epidemiological studies: pesticide exposure as a risk factor for ALS. Environ Res 2012;117:112-119.
29 Capozzella A, Sacco C, Chighine A, Loreti B, Scala B, Casale T, et al: Work related etiology of amyotrophic lateral sclerosis (ALS): a meta-analysis. Ann Ig 2014;26:456-472.

30 Kamel F, Umbach DM, Bedlack RS, Richards M, Watson M, Alavanja MC, et al: Pesticide exposure and amyotrophic lateral sclerosis. Neurotoxicology 2012;33:457-462.

31 Sutedja NA, Veldink JH, Fischer K, Kromhout $\mathrm{H}$, Heederik D, Huisman $\mathrm{MH}$, et al: Exposure to chemicals and metals and risk of amyotrophic lateral sclerosis: a systematic review. Amyotroph Lateral Scler 2009;10:302309.

32 Kang H, Cha ES, Choi GJ, Lee WJ: Amyotrophic lateral sclerosis and agricultural environments: a systematic review. J Korean Med Sci 2014;29:1610-1617.

33 Wang MD, Gomes J, Cashman NR, Little J, Krewski D: A meta-analysis of observational studies of the association between chronic occupational exposure to lead and amyotrophic lateral sclerosis. J Occup Environ Med 2014; 56:1235-1242.

34 Fitzgerald KC, O’Reilly EJ, Falcone GJ, McCullough ML, Park Y, Kolonel LN, et al: Dietary $\omega-3$ polyunsaturated fatty acid intake and risk for amyotrophic lateral sclerosis. JAMA Neurol 2014;71:1102-1110.

35 Abraham A, Drory VE: Influence of serum uric acid levels on prognosis and survival in amyotrophic lateral sclerosis: a meta-analysis. J Neurol 2014;261:1133-1138.

36 Huang R, Guo X, Chen X, Zheng Z, Wei Q Cao B, et al: The serum lipid profiles of amyotrophic lateral sclerosis patients: a study from south-west China and a meta-analysis. Amyotroph Lateral Scler Frontotemporal Degener 2015;16:359-365

37 Tsilidis KK, Kasimis JC, Lopez DS, Ntzani EE, Ioannidis JP: Type 2 diabetes and cancer: umbrella review of meta-analyses of observational studies. BMJ 2015;350:g7607.

38 Belbasis L, Panagiotou OA, Dosis V, Evangelou E: A systematic appraisal of field synopses in genetic epidemiology: a HuGE review. Am J Epidemiol 2015;181:1-16.

39 Fang F, Kwee LC, Allen KD, Umbach DM, Ye $\mathrm{W}$, Watson $\mathrm{M}$, et al: Association between blood lead and the risk of amyotrophic lateral sclerosis. Am J Epidemiol 2010;171:11261133.

40 Kamel F, Umbach DM, Munsat TL, Shefner JM, Hu H, Sandler DP: Lead exposure and amyotrophic lateral sclerosis. Epidemiology 2002;13:311-319.

41 Callaghan B, Feldman D, Gruis K, Feldman E: The association of exposure to lead, mercury, and selenium and the development of amyotrophic lateral sclerosis and the epigenetic implications. Neurodegener Dis 2011;8:1-8.
42 Chen H, Richard M, Sandler DP, Umbach DM, Kamel F: Head injury and amyotrophic lateral sclerosis. Am J Epidemiol 2007;166: 810-816.

43 Jafari S, Etminan M, Aminzadeh F, Samii A: Head injury and risk of Parkinson disease: a systematic review and meta-analysis. Mov Disord 2013;28:1222-1229.

44 Wirdefeldt K, Adami HO, Cole P, Trichopoulos D, Mandel J: Epidemiology and etiology of Parkinson's disease: a review of the evidence. Eur J Epidemiol 2011;26(suppl 1):S1-S58.

45 Armon C, Nelson LM: Is head trauma a risk factor for amyotrophic lateral sclerosis? An evidence based review. Amyotroph Lateral Scler 2012;13:351-356.

46 Tsilidis KK, Papatheodorou SI, Evangelou E, Ioannidis JP: Evaluation of excess statistical significance in meta-analyses of 98 biomarker associations with cancer risk. J Natl Cancer Inst 2012;104:1867-1878.

47 Tzoulaki I, Siontis KC, Evangelou E, Ioannidis JP: Bias in associations of emerging biomarkers with cardiovascular disease. JAMA Intern Med 2013;173:664-671.

48 Shea BJ, Hamel C, Wells GA, Bouter LM, Kristjansson E, Grimshaw J, et al: AMSTAR is a reliable and valid measurement tool to assess the methodological quality of systematic reviews. J Clin Epidemiol 2009;62:1013-1020.

49 Shea BJ, Grimshaw JM, Wells GA, Boers M, Andersson N, Hamel C, et al: Development of AMSTAR: a measurement tool to assess the methodological quality of systematic reviews. BMC Med Res Methodol 2007;7:10.

50 von Elm E, Altman DG, Egger M, Pocock SJ, Gøtzsche PC, Vandenbroucke JP, et al: The strengthening the reporting of observational studies in epidemiology (STROBE) statement: guidelines for reporting observational studies. J Clin Epidemiol 2008;61:344-349.

51 Ioannidis JP: The importance of potential studies that have not existed and registration of observational data sets. JAMA 2012;308: 575-576.

52 Dal-Ré R, Ioannidis JP, Bracken MB, Buffler PA, Chan AW, Franco EL, et al: Making prospective registration of observational research a reality. Sci Transl Med 2014;6:224cm1.

53 Fitzgerald KC, O’Reilly ÉJ, Fondell E, Falcone GJ, McCullough ML, Park Y, et al: Intakes of vitamin $\mathrm{C}$ and carotenoids and risk of amyotrophic lateral sclerosis: pooled results from 5 cohort studies. Ann Neurol 2013;73:236-245.

54 Zheng Z, Sheng L, Shang H: Statins and amyotrophic lateral sclerosis: a systematic review and meta-analysis. Amyotroph Lateral Scler Frontotemporal Degener 2013;14:241-245.

55 Zhou H, Chen G, Chen C, Yu Y, Xu Z: Association between extremely low-frequency electromagnetic fields occupations and amyotrophic lateral sclerosis: a meta-analysis. PLoS One 2012;7:e48354. 